\title{
Nonlocal annihilation of Weyl fermions in correlated systems
}

\author{
L. Crippa $\odot,{ }^{1}$ A. Amaricci, ${ }^{2,1}$ N. Wagner,${ }^{3}$ G. Sangiovanni, ${ }^{3}$ J. C. Budich,${ }^{4}$ and M. Capone ${ }^{1}$ \\ ${ }^{1}$ Scuola Internazionale Superiore di Studi Avanzati (SISSA), Via Bonomea 265, 34136 Trieste, Italy \\ ${ }^{2}$ CNR-IOM DEMOCRITOS, Istituto Officina dei Materiali, Consiglio Nazionale delle Ricerche, Via Bonomea 265, I-34136 Trieste, Italy \\ ${ }^{3}$ Institut für Theoretische Physik und Astrophysik and Würzburg-Dresden Cluster of Excellence ct.qmat, \\ Universität Würzburg, 97074 Würzburg, Germany \\ ${ }^{4}$ Institute of Theoretical Physics, Technische Universität Dresden, 01062 Dresden, Germany
}

(Received 26 April 2019; revised manuscript received 5 November 2019; published 22 January 2020)

\begin{abstract}
Weyl semimetals (WSMs) are characterized by topologically stable pairs of nodal points in the band structure that typically originate from splitting a degenerate Dirac point by breaking symmetries such as time-reversal or inversion symmetry. Within the independent-electron approximation, the transition between an insulating state and a WSM requires the local creation or annihilation of one or several pairs of Weyl nodes in reciprocal space. Here, we show that strong electron-electron interactions may qualitatively change this scenario. In particular, we reveal that the transition to a Weyl semimetallic phase can become discontinuous, and, quite remarkably, pairs of Weyl nodes with a finite distance in momentum space suddenly appear or disappear in the spectral function. We associate this behavior with the buildup of strong many-body correlations in the topologically nontrivial regions, manifesting in dynamical fluctuations in the orbital channel. We also highlight the impact of electronic correlations on the Fermi arcs.
\end{abstract}

DOI: 10.1103/PhysRevResearch.2.012023

The classification of topological states of matter has revolutionized our understanding of insulating [1] and nodal materials [2-4], leading to a novel conceptual framework for band structure theory. Regarding insulating systems, topological insulators (TIs) [5-9] represent a paradigmatic example of topological states, which are protected by symmetries such as time-reversal symmetry (TRS), and are distinguished from conventional band insulators by topological band structure invariants. Within the independent-electron approximation, phase transitions between topologically distinct insulators occur via semimetallic Dirac points.

In three spatial dimensions (3D), such critical Dirac points may be promoted to extended Weyl semimetal (WSM) phases [10-12] upon breaking TRS or inversion symmetry, since isolated nodal points in the band structure are topologically stable in 3D. Concretely, a symmetry-breaking perturbation splits conventional spin-degenerate Dirac cones into pairs of nondegenerate Weyl fermions with opposite chirality [see Fig. 1(a) for an illustration]. At least within the independentelectron approximation such Weyl nodes can only be locally annihilated by continuously bringing them together in reciprocal space with their chiral antidotes [10], and the robustness of WSMs against interactions as well as various intriguing correlation effects have recently been reported [13-21]. WSMs exhibit fascinating transport phenomena, related to the chiral anomaly and relativistic electronic dispersion at low energy,

Published by the American Physical Society under the terms of the Creative Commons Attribution 4.0 International license. Further distribution of this work must maintain attribution to the author(s) and the published article's title, journal citation, and DOI. and have recently become a major focus of theoretical and experimental research, in a variety of materials ranging from pyrochlore iridates and tantalum monopnictides to synthetic materials such as optical lattices with laser-assisted tunneling [22-35].

In this work, we report on the discovery of drastic changes to the phenomenology of WSMs in the presence of strong electronic correlations [see Fig. 1(b) for a visualization]. We show this by solving a multiorbital 3D Hubbard model with a TRS-breaking term in the framework of dynamical mean-field theory (DMFT) [36]. Quite remarkably, we find that interactions may lead to the discontinuous annihilation of Weyl nodes in momentum space at a first-order WSMto-insulator transition. In this scenario, pairs of Weyl points suddenly disappear notwithstanding their finite separation in momentum space, leaving behind a gapped state. This genuine many-body effect may have a deep impact on the prediction and possible detection of WSMs in correlated materials. Conversely, when approaching the transition to a WSM from the trivial band insulator (BI) phase, the nodal phase is entered discontinuously in the strongly interacting regime, without the continuous bifurcation of a degenerate Dirac cone into two distinct Weyl nodes. Furthermore, we unveil the presence of a quantum tricritical point (QTP), associated with an instability in the orbital channel. Beyond this point the WSM region is dramatically reduced and eventually suppressed in favor of a discontinuous transition between two insulators.

We consider a 3D simple cubic lattice model of spinful electrons with two orbitals per site and unit lattice constant, describing a 3D TI exposed to a TRS-breaking perturbation. The Hamiltonian $H$ may be written as the sum of a single-particle term diagonal in momentum space and a two-body interaction local in real space $H=\sum_{\mathbf{k}} \Psi_{\mathbf{k}}^{\dagger} H_{\mathbf{k}} \Psi_{\mathbf{k}}+$ 
(a)

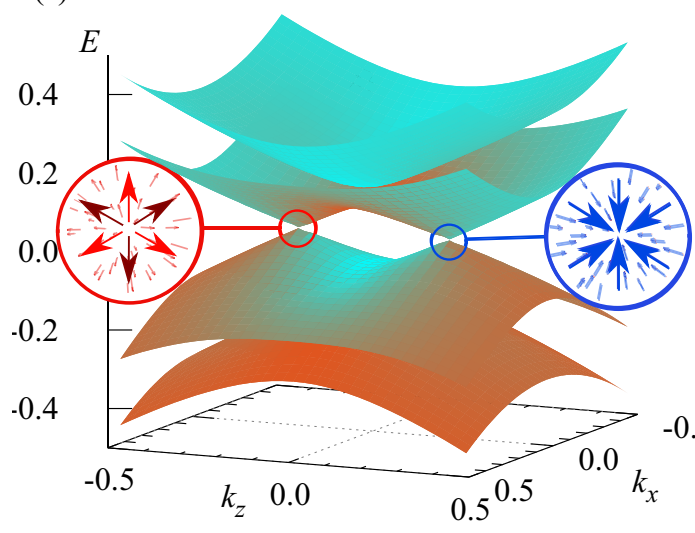

(b)

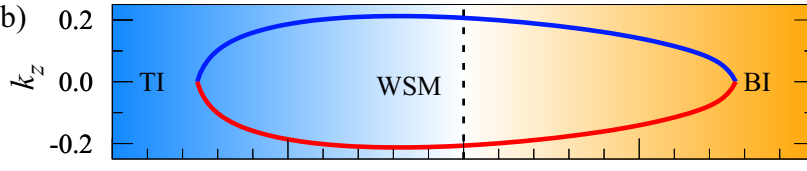

(c) 0.2

$+N^{N} 0.0$

-0.2
0.2
$\mathrm{~N}^{\mathrm{N}} 0.0$
-0.2

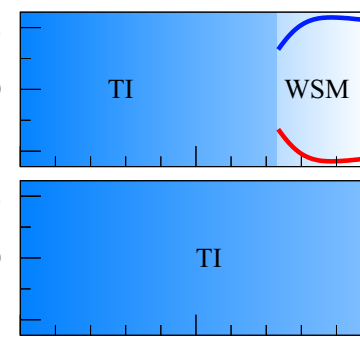

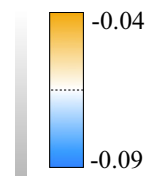

$-0.10$

$-0.05$

FIG. 1. (a) Band structure of (1) in the WSM phase on $k_{y}=0$ plane including the $\Gamma$ point (orbital character in color). The circular insets show the Berry flux near the two Weyl points. (b)-(d) Position of Weyl nodes along $k_{z}$ (for $k_{x}=k_{y}=0$ ) as a function of $M-M_{0}$ for increasing values of $U$ and $b_{z}=0.1$. Here $M_{0}$ is determined by the condition $M+\operatorname{Tr}\left[\sigma_{0} \tau_{z} \Sigma(0)\right] / 4=3$, where $\Sigma(0)$ is the self-energy function at zero frequency. This condition, which in the case $b_{z}=0$ [37] determines the topological transition point, is used here to center and compare data at different interactions strengths. The background colors in the panels reflect the behavior of the correlation strength $\Xi$. For small $U(\approx 2.0)$ the Weyl points form or annihilate continuously (b) at $\Gamma$. At larger $U$ (c) they (dis)appear discontinuously at the boundary with an insulating state, while the width of the WSM phase reduces. Eventually (d), for large $U(>5.7)$ the WSM region disappears and the system undergoes a direct first-order transition between strong TI and BI.

$\sum_{i} H_{i}^{\text {int }}$, where we introduced the four-component spinor $\Psi(\mathbf{k})=\left(c_{\mathbf{k} 1 \uparrow}, c_{\mathbf{k} 2 \uparrow}, c_{\mathbf{k} 1 \downarrow}, c_{\mathbf{k} 2 \downarrow}\right)$, with the operator $c_{\mathbf{k} \alpha \sigma}(\alpha=$ $1,2 ; \sigma=\uparrow, \downarrow)$ annihilating an electron with momentum $\mathbf{k}$ and spin $\sigma$ in orbital $\alpha$. The noninteracting term is

$$
\begin{aligned}
H_{\mathbf{k}}= & \mathcal{M}(\mathbf{k}) \sigma_{0} \tau_{z}+b_{z} \sigma_{z} \tau_{z} \\
& +\lambda\left(\sin \left(k_{x}\right) \sigma_{z} \tau_{x}-\sin \left(k_{y}\right) \sigma_{0} \tau_{y}+\sin \left(k_{z}\right) \sigma_{x} \tau_{x}\right),
\end{aligned}
$$

where $\sigma_{i}$ and $\tau_{j}$ with $i, j=x, y, z$ denote Pauli matrices in spin and orbital space, respectively, and $\sigma_{i} \tau_{j}$ are Kronecker products. The dispersion is $\mathcal{M}(\mathbf{k})=M-\epsilon\left(\cos k_{x}+\cos k_{y}+\right.$ $\cos k_{z}$ ) and $b_{z}$ is the strength of the TRS-breaking term. In the following we will consider $\epsilon$ as our unit of energy and fix $\lambda$ to 0.5 .

In the noninteracting regime and for $b_{z}=0$ the model describes a weak TI (WTI) and a strong TI (STI) for $M<1$ and $1<M<3$, respectively, and a trivial BI for $M>3$. The topological transitions between these phases occur via the continuous closure of the energy gap and the concomitant formation of a degenerate Dirac cone [37,38]. A finite value of $b_{z}$ breaks the TRS and lifts the spin degeneracy, though without giving rise to a net magnetization. The Dirac cone at the transition point splits into two Weyl cones separated in momentum space along $k_{z}$. Each Weyl node acts as the magnetic (anti)monopole for the Berry phase and is characterized by a Chern invariant measuring its chirality $[1,39,40]$. The absence of a protecting TRS makes the topological character of the WSM in some sense more robust than the usual symmetry-protected TIs. In the absence of interaction the destruction (formation) of the Weyl cones can only occur through their continuous annihilation (separation) at a specific high-symmetry point.

The topological quantum phase transition points are thus replaced by two distinct WSM phases in two windows $\Delta M=$ $2 b_{z}$ centered around $M=1$ and $M=3$. The one separating the WTI from the STI features three distinct couples of Weyl points, located near the $X, X^{\prime}$, and the $\Gamma$ points and separated along the $k_{z}$ direction in the reciprocal lattice space. The second WSM state, separating the STI from the BI, features only one pair of Weyl nodes, located along $k_{z}$ around $\Gamma$; see Fig. 1(a).

In the Wannier basis, the Coulomb interaction is described by a set of parameters as the on-site intraorbital and interorbital terms $\left(U\right.$ and $\left.U^{\prime}\right)$ and the Hund term $J$. The couplings are related by $U^{\prime}=U-2 J$ for electrons with opposite spin in the two orbitals and $U^{\prime \prime}=U-3 J$ for electrons with the same spin. The interaction term reads

$$
\begin{aligned}
H_{\mathrm{int}}= & U \sum_{i l} n_{i l \uparrow} n_{i l \downarrow} \\
& +(U-2 J) \sum_{i l \neq l^{\prime}} n_{i l \uparrow} n_{i l^{\prime} \downarrow} \\
& +(U-3 J) \sum_{i l \neq l^{\prime}}\left(n_{i l \uparrow} n_{i l^{\prime} \uparrow}+n_{i l \downarrow} n_{i l^{\prime} \downarrow}\right) .
\end{aligned}
$$

We found that the features of the topological phase transitions are robust across a wide range of variations of the interaction parameters. Therefore, to keep the discussion well defined, in the rest of this work we will focus on the case $J=0, U^{\prime}=U$. In the Supplemental Material [41] we present additional results with the effects of the Hund coupling $J$ and of the $U^{\prime} / U$ ratio on the topological phase transition.

The interacting model is solved nonperturbatively by means of DMFT [36-38], i.e., by mapping the lattice problem onto a single-site quantum impurity coupled to an effective bath which is self-consistently determined. Within the DMFT approach, the effects of the interaction are contained in the local self-energy function $\Sigma$, which in our case is a $4 \times 4$ matrix in the spin and orbital space retaining the local symmetries of the problem. We obtain zero-temperature results using a 
Lanczos-based exact-diagonalization method $[36,42,43]$ discretizing the bath with $N_{b}=9$ levels. The main result of this work, namely the nonlocal annihilation of the Weyl points in the presence of electronic interaction, is robust against a change of $N_{b}$ as well as for finite temperatures up to a scale on the order of one-tenth of the energy unit. We checked the stability of our findings comparing with a continuous-time quantum Monte Carlo solver [44].

The two aforementioned WSM phases are found to be influenced by the local Coulomb repulsion in a qualitatively distinct way. The one occurring between the weak and strong TIs undergoes a rather predictable and basically adiabatic evolution as a function of $U$. By contrast, the WSM separating the STI and the BI drastically changes its nature as the electron-electron interaction exceeds a certain value. This main result of our work is illustrated in Figs. 1(b)-1(d). The position of the Weyl points in $k_{z}$ as a function of $M$ is shown by the red and blue lines. In summary, for small $U$ [Fig. 1(b)] the two nodes form a closed path, bridging the STI with the BI region across the vertical dotted line at which, in the absence of $b_{z}$, the topological phase transition occurs. This continuous behavior of the Weyl nodes is adiabatically connected with the noninteracting case.

Surprisingly, for intermediate values of the interaction strength [Fig. 1(c)] a discontinuous evolution is observed. The Weyl nodes appear all of a sudden already separated in $k$ space at a critical value of the mass term $M$. Upon further increasing $M$, the position of the cones evolves continuously, but they soon nonlocally disappear in favor of the band insulator, without meeting in momentum space. Correspondingly, the red and blue lines are no longer connected, a situation that is fundamentally forbidden within the independent-electron approximation.

This intriguing phenomenon can be further characterized by inspecting the correlation strength $\Xi$, which we measure in terms of the deviation from Hartree-Fock theory of our semimetallic solutions $[37,38,45]$. Since within DMFT we take into account contributions to the (local) self-energy to all orders, our solution displays self-energy corrections that are much richer than Hartree-Fock theory. In particular, the self-energy $\Sigma$ is found to exhibit a pronounced frequency structure at intermediate-to-large $U$, thus qualitatively deviating from the static mean-field solution [46,47]. This is a consequence of the pronounced many-body fluctuations of the STI and of the correlated Weyl phases, originating from their orbitally entangled nature. We can quantify this by computing the difference between the low-frequency limit, which describes the quasiparticles, and the high-energy limit $\Xi=\operatorname{Tr}\left[\sigma_{0} \tau_{z} \Sigma(0)-\sigma_{0} \tau_{z} \Sigma^{\mathrm{HF}}\right] / \operatorname{Tr}\left[\sigma_{0} \tau_{z} \Sigma(0)\right]$, where $\Sigma^{\mathrm{HF}}$ is the limit of $\Sigma$ at high frequency: here the $\omega$ dependence is hardly visible and $\Sigma$ can be seen as that of a static HartreeFock-like mean field $[37,38]$. The behavior of $\Xi$ is represented by the background color. In Fig. 1(b) the evolution of $\Xi$ as a function of $M$ is smooth everywhere and, similarly to the noninteracting case, the Weyl nodes continuously meet in $k$ space at each insulator-to-semimetal transition. In Fig. 1(c), the correlation strength changes discontinuously at specific values of $M$ at both sides of the dashed line, signaling the abrupt occurrence of the WSM phase from both directions. Since the electron-electron interaction suppresses charge fluctuations associated with the formation of Weyl nodes, the WSM region generally shrinks as $U$ increases.

For even larger interaction strength [Fig. 1(d)], the system undergoes a discontinuous transition directly from the strong TI to the trivial BI without the semimetal in between. In this regime, the character of the WSM would be so strongly modified by many-body effects that the most natural evolution for the system is to link the two distinct insulating phases without any intermediate semimetal. This is evident from the sudden change of color at the critical value of $M$, which takes place with hardly any shading, as the semimetallic region is completely suppressed. To summarize, in the nontrivial regions the ground state develops a robust many-body character, encoded in a large (negative) value of $\Xi$, which cannot be continuously reconciled with the far less correlated nature of the solution in the trivial BI phase. This effect manifests in the discontinuous change of the (frequency dependent) self-energy. This behavior is reminiscent of the strongly correlated transition found in the presence of TRS and inversion symmetry, where a Dirac semimetal line (rather than a WSM region) cannot be continued to large values of $U[37,38]$.

There is a more intuitive way of interpreting our results. Concerning the topological nature of the system, the solution of the interacting problem can be recast into a quadratic effective Hamiltonian containing all the terms of Eq. (1). This way the effect of the electron-electron interaction is accounted for by renormalized parameters whose evolution can be compared to the noninteracting case. The two relevant indicators are the effective mass $M^{\text {eff }}=M+\operatorname{Tr}\left[\sigma_{0} \tau_{z} \Sigma(0)\right] / 4$ and the effective TRS-breaking field $b_{z}^{\text {eff }}=b_{z}+\operatorname{Tr}\left[\sigma_{z} \tau_{z} \Sigma(0)\right] / 4$. $M^{\text {eff }}$ controls the renormalized energy separation between the orbitals, while $b_{z}^{\text {eff }}$ corresponds to the effective lifting of the spin degeneracy. The behavior of these two quantities is displayed in Fig. 2 as a function of the bare mass term $M-M_{0}$, where

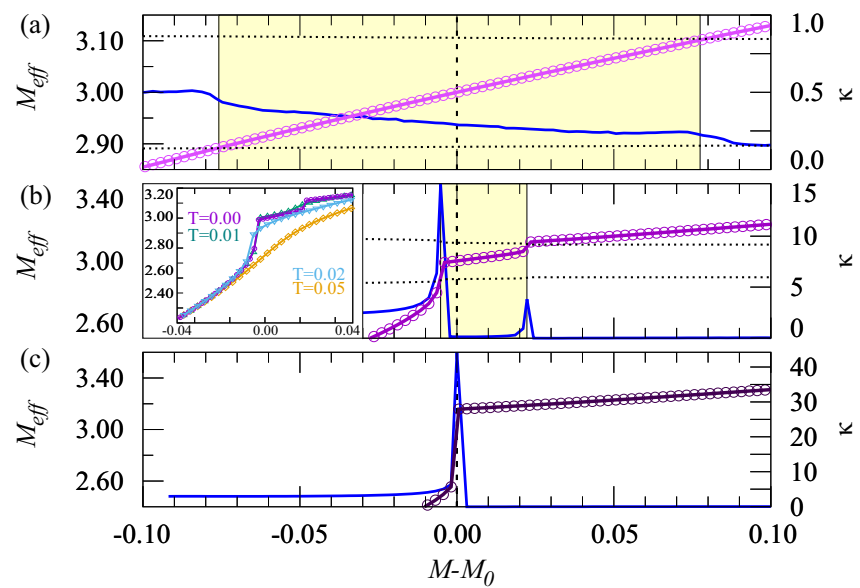

FIG. 2. $M^{\text {eff }}$ behavior as a function of the bare mass for different values of interaction: (a) $U=3.0$, (b) $U=5.5$, (c) $U=5.9$. $M_{0}$ is defined as in Fig. 1. The shaded regions correspond to the WSM, that of the noninteracting system being the full width of the panels. The dotted lines represent (where significant) the expected width of the WSM region, spanning the interval $M^{\text {eff }}=\left[3-b_{z}^{\text {eff }}, 3+b_{z}^{\text {eff }}\right]$. The blue lines represent the orbital compressibility $\kappa$. The inset to panel (b) shows the behavior of $M^{\mathrm{eff}}$ for $U=5.5$ as a function of temperature. 


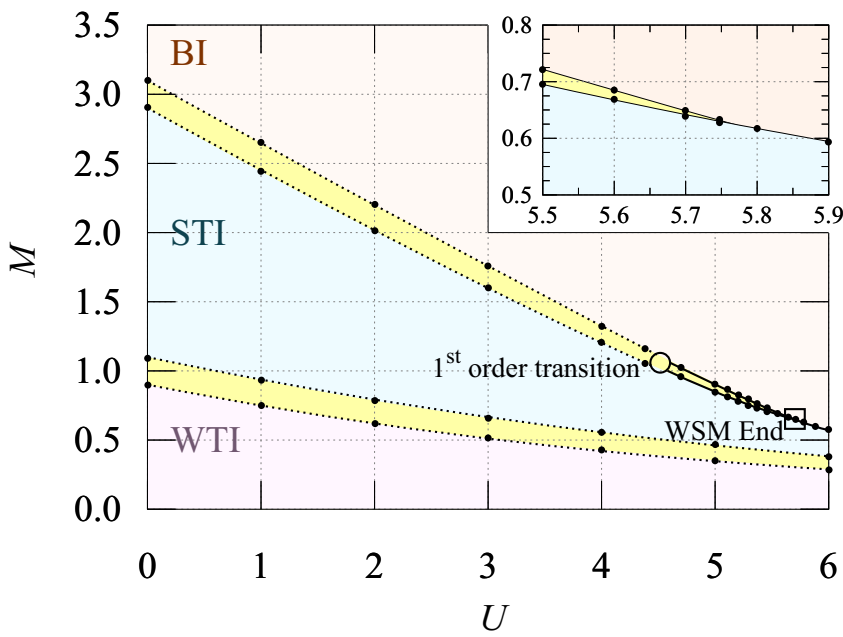

FIG. 3. Phase diagram of the model for $b_{z}=0.1$. The regions (yellow) between the dotted lines are WSM. The inset shows the closing of the upper WSM region. Numerical results are represented by black symbols. At $U=0$, the widths of the semimetal regions are $2 b_{z}$, centered around $M^{\text {eff }}=1,3$. The first semimetal region remains open when increasing the interaction, and the transition from WTI to WSM to STI is always continuous. A second critical point signals the value of $U$ for which the upper WSM region closes.

$M_{0}$ is the critical value of $M$ for which $M^{\text {eff }}=3$, i.e., the condition to have a topological phase transition for $b_{z}=0$ [37]. In addition, we show the behavior of the orbital compressibility $\kappa=\partial T_{i}^{z} / \partial M$, where $T_{i}^{z}=\sum_{\sigma} n_{i 1 \sigma}-n_{i 2 \sigma}$ is the local orbital polarization. In analogy with the noninteracting model, the WSM region is characterized by $\left|M^{\text {eff }}-3\right| \leqslant b_{z}^{\text {eff }}$ and is marked in yellow.

In the weak-coupling regime [Fig. 2(a)] the effective mass evolves continuously across the boundary of the WSM phase. The orbital compressibility smoothly decreases upon approaching the BI region. On the contrary, in Fig. 2(b) the behavior of the effective parameters becomes discontinuous. In particular, the evolution of the effective mass term $M^{\text {eff }}$ exhibits pronounced discontinuities at the crossing of the $b_{z}^{\text {eff }}$ lines delimiting the semimetallic phase. The many-body nature of the strongly correlated solutions is demonstrated by the behavior of $\kappa$, displaying large peaks at the transition points. Interestingly, the WSM region extends asymmetrically around $M_{0}$. Indeed, the effect of the interaction is more pronounced in the strong-TI phase, because of the more fluctuating orbital polarization $T_{z}$, as compared to the close to fully orbitally polarized BI. In Fig. 2(c), we report the evolution of the effective parameters for a larger interaction strength $U$. In this regime, the Weyl region is entirely absent. $M^{\text {eff }}$ has a large discontinuity just around the critical value $M_{0}$, unveiling the direct first-order transition between the STI and the BI phases. This transition is characterized by a large peak of the orbital compressibility at the transition point. In the inset to Fig. 2(b) we show how the jumps in $M^{\text {eff }}$ evolve as a function of the temperature $T$. The temperature at which they disappear for $U=5.5$ is on the order of $1 / 20$ of our hopping energy.

An overview of our findings is provided in Fig. 3, where we show the complete phase diagram of the model in the $U-M$ plane. The different phases of the model are separated by the two WSM regions. The boundary lines have an overall decreasing behavior as a function of $U$, which results from the tendency of the interaction to favor the orbitally polarized states, i.e., the trivial BI with two electrons occupying the lowest orbital [41]. As anticipated above, the two WSM phases undergo a dramatically different evolution in the presence of interaction. The WSM region between WTI and STI is only slightly affected by the electron-electron repulsion. This region displays a minor reduction of its width and the transition lines keep their continuous character. On the other hand, the WSM region separating the STI and the BI keeps its continuous character only up to a critical value of the interaction $U_{c}=4.5$. Beyond this value the topological transition lines acquire a first-order character. The width of this WSM phase gradually decreases upon increasing the interaction as a consequence of the renormalization of the effective term $b_{z}^{\text {eff }}$. In the inset of Fig. 3 we show the evolution of the Weyl phase
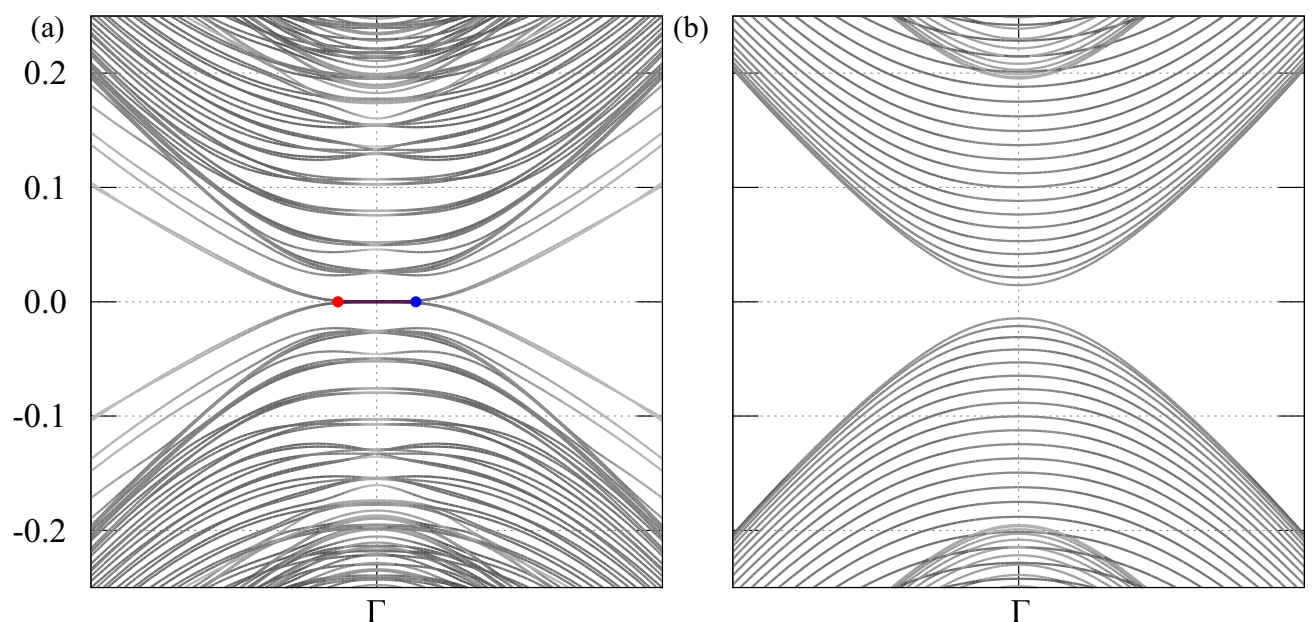

FIG. 4. Electronic structure of a slab geometry near the $\Gamma$ point at $U=5.6$. The two panels report the behavior for two different values of the mass term $M$ across the WSM to BI transition (see inset of Fig. 3). (a) $M=0.665$; the points (red and blue) indicate the Weyl nodes with opposite chirality. The band segment connecting them is the Fermi arc. (b) $M=0.670$; the system is a band insulator. 
boundary lines up to the closure of the corresponding interval for $U=5.75$.

Finally we investigate the fate of the boundary modes in the presence of correlations. A distinctive property of WSMs are Fermi arcs connecting the projection of the two bulk Weyl nodes onto the surface Brillouin zone. In a single-particle scheme, approaching the topological transition is associated with the progressive shortening of the Fermi arcs until the two ends coalesce in an insulator. It is natural to expect that the scenario we unveiled influences this picture.

To address the correlated boundary states, in Fig. 4 we show the spectrum of the model Eq. (1) in a slab geometry with open boundary conditions along $y$. Our results illustrate the sudden (dis)appearance of the Fermi arcs across the transition for a large value of the interaction $U$, reflecting the first-order character of the transition observed in the bulk system (see Fig. 3).

In conclusion, we revealed that in the presence of a large electron-electron interaction the transition to and from a WSM can become of first order. In this scenario, the strongly correlated Weyl nodes can appear or annihilate discontinuously in a nonlocal fashion in reciprocal space at the transition point. The change in the nature of the transition is inherently linked to a many-body nature of the correlated Weyl semimetal whose distinctive property is an enhancement of the orbital compressibility. We described this behavior by the evolution of effective model parameters. Finally, we showed that in the strongly interacting regime the WSM region can progressively close in favor of a direct transition between two gapped phases.

The authors thank B. Trauzettel for important comments and careful reading of the manuscript. A.A. and M.C. acknowledge support from the H2020 Framework Programme, under ERC Advanced Grant No. 692670 “FIRSTORM.” L.C., A.A., and M.C. acknowledge financial support from MIUR PRIN 2015 (Prot. 2015C5SEJJ001) and SISSA/CNR project "Superconductivity, Ferroelectricity, and Magnetism in Bad Metals" (Prot. 232/2015). J.C.B. acknowledges financial support from the German Research Foundation (DFG) through the Collaborative Research Centre SFB 1143. G.S. acknowledges financial support by the DFG through SFB 1170 "ToCoTronics" and the Würzburg-Dresden Cluster of Excellence on Complexity and Topology in Quantum Matter-ct.qmat (EXC 2147, Project ID 39085490). N.W. and G.S. gratefully acknowledge the Gauss Centre for Supercomputing e.V. [48] for funding this project by providing computing time on the GCS supercomputer SuperMUC at Leibniz Supercomputing Centre [49].
[1] X.-L. Qi and S.-C. Zhang, Topological insulators and superconductors, Rev. Mod. Phys. 83, 1057 (2011).

[2] L. M. Schoop, M. N. Ali, C. Straßer, A. Topp, A. Varykhalov, D. Marchenko, V. Duppel, S. S. P. Parkin, B. V. Lotsch, and Ch. R. Ast, Dirac cone protected by non-symmorphic symmetry and three-dimensional Dirac line node in $\mathrm{ZrSiS}$, Nat. Commun. 7, 11696 (2016).

[3] S. M. Young, S. Zaheer, J. C. Y. Teo, C. L. Kane, E. J. Mele, and A. M. Rappe, Dirac Semimetal in Three Dimensions, Phys. Rev. Lett. 108, 140405 (2012).

[4] S. M. Young and C. L. Kane, Dirac Semimetals in Two Dimensions, Phys. Rev. Lett. 115, 126803 (2015).

[5] C. L. Kane and E. J. Mele, $Z_{2}$ Topological Order and the Quantum Spin Hall Effect, Phys. Rev. Lett. 95, 146802 (2005).

[6] B. A. Bernevig, T. L. Hughes, and S.-C. Zhang, Quantum spin Hall effect and topological phase transition in $\mathrm{HgTe}$ quantum wells, Science 314, 1757 (2006).

[7] L. Fu, C. L. Kane, and E. J. Mele, Topological Insulators in Three Dimensions, Phys. Rev. Lett. 98, 106803 (2007).

[8] H. Zhang, C.-X. Liu, X.-L. Qi, Xi Dai, Z. Fang, and S.-C. Zhang, Topological insulators in $\mathrm{Bi}_{2} \mathrm{Se}_{3}, \mathrm{Bi}_{2} \mathrm{Te}_{3}$ and $\mathrm{Sb}_{2} \mathrm{Te}_{3}$ with a single Dirac cone on the surface, Nat. Phys. 5, 438 (2009).

[9] S. Ryu, A. P. Schnyder, A. Furusaki, and A. W. W. Ludwig, Topological insulators and superconductors: Tenfold way and dimensional hierarchy, New J. Phys. 12, 065010 (2010).

[10] S. Murakami, Phase transition between the quantum spin Hall and insulator phases in 3D: Emergence of a topological gapless phase, New J. Phys. 9, 356 (2007).
[11] X. Wan, A. M. Turner, A. Vishwanath, and S. Y. Savrasov, Topological semimetal and Fermi-arc surface states in the electronic structure of pyrochlore iridates, Phys. Rev. B 83, 205101 (2011).

[12] N. P. Armitage, E. J. Mele, and A. Vishwanath, Weyl and Dirac semimetals in three-dimensional solids, Rev. Mod. Phys. 90, 015001 (2018).

[13] W. Witczak-Krempa, M. Knap, and D. Abanin, Interacting Weyl Semimetals: Characterization Via the Topological Hamiltonian and Its Breakdown, Phys. Rev. Lett. 113, 136402 (2014).

[14] M. Laubach, C. Platt, R. Thomale, T. Neupert, and S. Rachel, Density wave instabilities and surface state evolution in interacting Weyl semimetals, Phys. Rev. B 94, 241102(R) (2016).

[15] B. Roy, P. Goswami, and V. Juričić, Interacting Weyl fermions: Phases, phase transitions, and global phase diagram, Phys. Rev. B 95, 201102(R) (2017).

[16] J. Carlström and E. J. Bergholtz, Symmetry-enforced stability of interacting Weyl and Dirac semimetals, Phys. Rev. B 97, 161102(R) (2018).

[17] T. Meng and J. C. Budich, Unpaired Weyl Nodes from LongRanged Interactions: Fate of Quantum Anomalies, Phys. Rev. Lett. 122, 046402 (2019).

[18] T. Meng, A. G. Grushin, K. Shtengel, and J. H. Bardarson, Theory of a 3+1D fractional chiral metal: Interacting variant of the Weyl semimetal, Phys. Rev. B 94, 155136 (2016).

[19] H.-H. Lai, S. E. Grefe, S. Paschen, and Q. Si, Weyl-Kondo semimetal in heavy-fermion systems, Proc. Natl. Acad. Sci. USA 115, 93 (2018). 
[20] H. Rostami, E. Cappelluti, and A. V. Balatsky, Helical metals and insulators: Sheet singularity of the inflated Berry monopole, Phys. Rev. B 98, 245114 (2018).

[21] T. Morimoto and N. Nagaosa, Weyl Mott insulator, Sci. Rep. 6, 19853 (2016).

[22] L. Lu, Z. Wang, D. Ye, L. Ran, L. Fu, J. D. Joannopoulos, and M. Soljačić, Experimental observation of Weyl points, Science 349, 622 (2015).

[23] L. Lu, L. Fu, J. D. Joannopoulos, and M. Soljacic, Weyl points and line nodes in gyroid photonic crystals, Nat. Photon. 7, 294 (2013).

[24] L. X. Yang, Z. K. Liu, Y. Sun, H. Peng, H. F. Yang, T. Zhang, B. Zhou, Y. Zhang, Y. F. Guo, M. Rahn, D. Prabhakaran, Z. Hussain, S.-K. Mo, C. Felser, B. Yan, and Y. L. Chen, Weyl semimetal phase in the non-centrosymmetric compound TaAs, Nat. Phys. 11, 728 (2015).

[25] S.-Y. Xu, I. Belopolski, N. Alidoust, M. Neupane, G. Bian, C. Zhang, R. Sankar, G. Chang, Z. Yuan, C.-C. Lee, S.-M. Huang, H. Zheng, J. Ma, D. S. Sanchez, B. Wang, A. Bansil, F. Chou, P. P. Shibayev, H. Lin, S. Jia, and M. Z. Hasan, Discovery of a Weyl fermion semimetal and topological Fermi arcs, Science 349, 613 (2015).

[26] S. Nakatsuji, N. Kiyohara, and T. Higo, Large anomalous Hall effect in a non-collinear antiferromagnet at room temperature, Nature (London) 527, 212 (2015).

[27] K. Deng, G. Wan, P. Deng, K. Zhang, S. Ding, E. Wang, M. Yan, H. Huang, H. Zhang, Z. Xu, J. Denlinger, A. Fedorov, H. Yang, W. Duan, H. Yao, Y. Wu, S. Fan, H. Zhang, X. Chen, and S. Zhou, Experimental observation of topological Fermi arcs in type-II Weyl semimetal $\mathrm{MoTe}_{2}$, Nat. Phys. 12, 1105 (2016).

[28] B. Q. Lv, N. Xu, H. M. Weng, J. Z. Ma, P. Richard, X. C. Huang, L. X. Zhao, G. F. Chen, C. E. Matt, F. Bisti, V. N. Strocov, J. Mesot, Z. Fang, X. Dai, T. Qian, M. Shi, and H. Ding, Observation of Weyl nodes in TaAs, Nat. Phys. 11, 724 (2015).

[29] M. Ikhlas, T. Tomita, T. Koretsune, M.-T. Suzuki, D. NishioHamane, R. Arita, Y. Otani, and S. Nakatsuji, Large anomalous Nernst effect at room temperature in a chiral antiferromagnet, Nat. Phys. 13, 1085 (2017).

[30] J. Jiang, Z. K. Liu, Y. Sun, H. F. Yang, C. R. Rajamathi, Y. P. Qi, L. X. Yang, C. Chen, H. Peng, C. C. Hwang, S. Z. Sun, S. K. Mo, I. Vobornik, J. Fujii, S. S. P. Parkin, C. Felser, B. H. Yan, and Y. L. Chen, Signature of type-II Weyl semimetal phase in $\mathrm{MoTe}_{2}$, Nat. Commun. 8, 1 (2017).

[31] K. Kuroda, T. Tomita, M. T. Suzuki, C. Bareille, A. A. Nugroho, P. Goswami, M. Ochi, M. Ikhlas, M. Nakayama, S. Akebi, R. Noguchi, R. Ishii, N. Inami, K. Ono, H. Kumigashira, A. Varykhalov, T. Muro, T. Koretsune, R. Arita, S. Shin, T. Kondo, and S. Nakatsuji, Evidence for magnetic Weyl fermions in a correlated metal, Nat. Mater. 16, 1090 (2017).

[32] B. Q. Lv, H. M. Weng, B. B. Fu, X. P. Wang, H. Miao, J. Ma, P. Richard, X. C. Huang, L. X. Zhao, G. F. Chen, Z. Fang, X. Dai, T. Qian, and H. Ding, Experimental Discovery of Weyl Semimetal TaAs, Phys. Rev. X 5, 031013 (2015).

[33] C.-H. Min, H. Bentmann, J. N. Neu, P. Eck, S. Moser, T. Figgemeier, M. Ünzelmann, K. Kissner, P. Lutz, R. J.
Koch, C. Jozwiak, A. Bostwick, E. Rotenberg, R. Thomale, G. Sangiovanni, T. Siegrist, D. Di Sante, and F. Reinert, Orbital Fingerprint of Topological Fermi Arcs in the Weyl Semimetal TaP, Phys. Rev. Lett. 122, 116402 (2019).

[34] S.-M. Huang, S.-Y. Xu, I. Belopolski, C.-C. Lee, G. Chang, B. Wang, N. Alidoust, G. Bian, M. Neupane, C. Zhang, S. Jia, A. Bansil, H. Lin, and M. Z. Hasan, A Weyl fermion semimetal with surface Fermi arcs in the transition metal monopnictide TaAs class, Nat. Commun. 6, 7373 (2015).

[35] T. Ohtsuki, Z. Tian, A. Endo, M. Halim, S. Katsumoto, Y. Kohama, K. Kindo, M. Lippmaa, and S. Nakatsuji, Straininduced spontaneous Hall effect in an epitaxial thin film of a Luttinger semimetal, Proc. Natl. Acad. Sci. USA 116, 8803 (2019).

[36] A. Georges, G. Kotliar, W. Krauth, and M. J. Rozenberg, Dynamical mean-field theory of strongly correlated fermion systems and the limit of infinite dimensions, Rev. Mod. Phys. 68, 13 (1996).

[37] A. Amaricci, J. C. Budich, M. Capone, B. Trauzettel, and G. Sangiovanni, First-Order Character and Observable Signatures of Topological Quantum Phase Transitions, Phys. Rev. Lett. 114, 185701 (2015).

[38] A. Amaricci, J. C. Budich, M. Capone, B. Trauzettel, and G. Sangiovanni, Strong correlation effects on topological quantum phase transitions in three dimensions, Phys. Rev. B 93, 235112 (2016).

[39] Z. Wang, X.-L. Qi, and S.-C. Zhang, Topological Order Parameters for Interacting Topological Insulators, Phys. Rev. Lett. 105, 256803 (2010).

[40] Z. Wang and S.-C. Zhang, Simplified Topological Invariants for Interacting Insulators, Phys. Rev. X 2, 031008 (2012).

[41] See Supplemental Material at http://link.aps.org/supplemental/ 10.1103/PhysRevResearch.2.012023 for a description of the effects of the parameters on the transition and the self-energy.

[42] M. Capone, L. de' Medici, and A. Georges, Solving the dynamical mean-field theory at very low temperatures using the Lanczos exact diagonalization, Phys. Rev. B 76, 245116 (2007).

[43] C. Weber, A. Amaricci, M. Capone, and P. B. Littlewood, Augmented hybrid exact-diagonalization solver for dynamical mean field theory, Phys. Rev. B 86, 115136 (2012).

[44] M. Wallerberger, A. Hausoel, P. Gunacker, A. Kowalski, N. Parragh, F. Goth, K. Held, and G. Sangiovanni, w2dynamics: Local one- and two-particle quantities from dynamical mean field theory, Comput. Phys. Commun. 235, 388 (2019).

[45] A. Amaricci, L. Privitera, F. Petocchi, M. Capone, G. Sangiovanni, and B. Trauzettel, Edge state reconstruction from strong correlations in quantum spin Hall insulators, Phys. Rev. B 95, 205120 (2017).

[46] J. C. Budich, R. Thomale, G. Li, M. Laubach, and S.-C. Zhang, Fluctuation-induced topological quantum phase transitions in quantum spin-Hall and anomalous-Hall insulators, Phys. Rev. B 86, 201407(R) (2012).

[47] J. C. Budich, B. Trauzettel, and G. Sangiovanni, Fluctuationdriven topological Hund insulators, Phys. Rev. B 87, 235104 (2013).

[48] See http://www.gauss-centre.eu.

[49] See http://www.lrz.de. 